\title{
Investigation into the Impact of Mutual Coupling on the Performance of a Multiuser MIMO System Employing Generalized Channel Inversion Technique
}

\author{
Feng Wang, Konstanty Bialkowski, and Marek E. Bialkowski \\ School of Information Technology and Electrical Engineering, The University of Queensland, Brisbane, QLD 4072, Australia \\ Correspondence should be addressed to Feng Wang, fwang@itee.uq.edu.au
}

Received 1 May 2011; Revised 21 June 2011; Accepted 21 June 2011

Academic Editor: Hon Tat Hui

Copyright ( 2011 Feng Wang et al. This is an open access article distributed under the Creative Commons Attribution License, which permits unrestricted use, distribution, and reproduction in any medium, provided the original work is properly cited.

\begin{abstract}
In this paper, the performance of generalized channel inversion (GCI) technique for a multiuser MIMO system operating under antenna mutual coupling conditions is investigated. The investigation is performed via theoretical derivations and computer simulations. The theoretical derivations show that the presence of mutual coupling may result in an increased performance in terms of sum rate capacity. The simulation experiments confirm the theoretical findings in examples of three groups of numerical results. These results show that the existence of mutual coupling in an array antenna at base station (BS) results in an increased sum rate capacity when antenna interelement spacing at BS is smaller than $0.55 \lambda$. Also it is shown that the presence of mutual coupling at mobile user (MS) terminals results in an increased sum rate capacity when antenna inter-element spacing at MSs is smaller than $0.4 \lambda$. When mutual coupling is considered at both BS and MSs, the system configuration with antenna inter-element spacing equal to $0.45 \lambda$ at BS and $0.4 \lambda$ at MSs leads to the highest sum rate capacity.
\end{abstract}

\section{Introduction}

Multiple-input multiple-output (MIMO) technology has received enormous interest due to its potential in providing high and robust data transmission for wireless communications $[1,2]$. Because of these attributes it is widely accepted as key technology for the next generation wireless systems $[3,4]$. Recent years have shown a significant shift of research attention from point-to-point to the multiuser MIMO systems [5], where a base station (BS) is communicating with a number of mobile stations (MSs). Recent works in [68] have shown that the advantages of employing multiple antennas in point-to-point systems can be translated to large gains in multiuser scenarios.

Research interests in multiuser MIMO systems have mainly focused on uplink multiple-access channel (MAC) and downlink broadcasting channel (BC). In this respect, the optimal encoding and decoding strategies for a MIMO MAC have been broadly studied. The information theory hints that the broadcasting case is by far the most challenging. In this case, an interuser interference occurs due to the spatially multiplexed transmitted signals at BS. It has been shown via theoretical derivations that the dirty paper coding (DPC) [9] is capable of achieving the capacity region [8] by precancelling the interuser interference at BS. However, the practical implementation of DPC in multiuser MIMO systems creates a considerable challenge due to its high computational burden of successive nonlinear coding and decoding. In order to ease these difficulties, DPC can be approximately implemented using the Tomlinson-Harashima (TH) precoding technique [10]. Unfortunately, the TH precoding technique still faces a considerable computational complexity at BS and MSs.

To overcome the hurdle associated with the computing intensive nonlinear precoding techniques, linear precoding techniques have been investigated for use in multiuser MIMO systems. Block diagonalization (BD) [11] is one of them and has received significant attention $[12,13]$ due to its simplicity and good performance. In $\mathrm{BD}$, the precoded signal of a MS lies in the null space of the other MSs' channels to prevent an inter-user interference. $\mathrm{BD}$ ignores the issues of noise level, and its performance is normally limited by 
the number of MSs and channel conditions experienced by the users. To increase the signal-to-interference-plus-noise (SINR) at each MS, an MMSE-based generalized channel inversion (GCI) technique is proposed in [14]. Opposite to $\mathrm{BD}$, this technique takes the noise into account in finding each MSs' precoding matrix. It provides an improved performance over the traditional BD.

The investigations concerning GCI have been presented for the case of simplified operation of array antennas at BS and MSs. In this case, the antenna elements are assumed to operate in an ideal manner with mutual coupling neglected. In practice, physical limitations in mobile terminals as well as in BS force antenna elements in an array to be placed with a small interelement spacing. This requirement is governed by compact MIMO transceivers especially on the user side. The resulting electromagnetic interactions between the antenna elements affect the performance of MIMO links. The effect of mutual coupling on array signal processing has been investigated in many works, such as $[15,16]$. In the context of point-to-point MIMO communications, studies on the effect of mutual coupling have been presented in [17-19]. More recently, mutual coupling has also been taken into account while investigating performance of multiuser MIMO systems $[13,20]$.

In this paper, the impact of antenna mutual coupling is investigated when a multiuser MIMO system employs a generalized channel inversion approach for its operation. The investigations include a theoretical analysis and computer simulations.

The paper is structured as follows. Section 2 presents the signal model for a multiuser MIMO system. Section 3 is devoted to modelling of electromagnetic interactions between the array elements and scatterers that are present in the propagation environment. Section 4 gives details of the generalized channel inversion algorithm that accounts for the effect of mutual coupling. Section 5 quantifies the effect of mutual coupling by presenting numerical results. Section 6 summarizes the findings of the undertaken research.

\section{Signal and Channel Model}

2.1. Signal Model for a Multiuser MIMO System. A narrowband downlink multiuser MIMO system with the BS serving $K \mathrm{MSs}$ is considered. It is assumed that the BS has $N$ transmit antennas. The $k$ th MS employs $M_{k}$ receive antennas. Assuming $\mathbf{x}_{k}$ denotes the $N_{k}$-dimensional transmitted symbol intended for the $k$ th MS and $\mathbf{w}_{k}$ is the $N \times N_{k}$ beamforming matrix for the $k$ th MS, the overall transmitted signal is given by

$$
\mathbf{x}=\sum_{k=1}^{K} \mathbf{w}_{k} \mathbf{x}_{k}
$$

Under the assumption of narrow-band MIMO system operation, the received signal at the $k$ th MS is expressed as

$$
\mathbf{y}_{k}=\mathbf{H}_{k} \mathbf{x}+\boldsymbol{\eta}_{k}=\mathbf{H}_{k} \mathbf{w}_{k} \mathbf{x}_{k}+\mathbf{H}_{k} \sum_{j \neq k} \mathbf{w}_{j} \mathbf{x}_{j}+\boldsymbol{\eta}_{k} .
$$

where $\mathbf{H}_{k}$ is a complex matrix representing the wireless MIMO channel between the BS and the $k$ th MS. $\boldsymbol{\eta}_{k}$ is a spatially and temporally distributed white noise vector with entries having a zero mean and variance $\sigma_{n}^{2}$, as given by

$$
E\left(\boldsymbol{\eta}_{k} \boldsymbol{\eta}_{k}^{*}\right)=\sigma_{n}^{2} \mathbf{I}_{M_{k}} \times M_{k} .
$$

2.2. Channel Model. In (2), $\mathbf{H}_{k}$ represents the wireless MIMO channel gain between the BS and the $k$ th MS. The $(p, q)$ th element in $\mathbf{H}_{k}$ is the fading transmission coefficient for the path between the $q$ th transmit antenna at BS and $p$ th receive antenna at the $k$ th $\mathrm{MS}$. The spatial-temporal properties of $\mathbf{H}_{k}$ are influenced by the transmitting and receiving antenna arrays' physical parameters in addition to the signal propagation environment. Here, it is postulated that the arrays at BS and MSs are formed by parallel linear wire dipoles. The BS and MSs are assumed to be surrounded by a large number of scattering objects distributed within a "circle of influence" [21]. It is also assumed that the links between the BS and different MSs do not share the same scattering environment. As a result, the signal fading is independent for different MSs. In addition, the correlations at the transmitter and receiver sides are independent so that the channel matrix $\mathbf{H}_{k}$ can be represented as

$$
\mathbf{H}_{k}=\left(\mathbf{R}_{\mathrm{MS}}^{k}\right)^{1 / 2} \mathbf{G}_{H}^{k} \mathbf{R}_{\mathrm{BS}}^{1 / 2},
$$

where $\mathbf{G}_{H}^{k}$ is a matrix with i.i.d. Gaussian entries with zero mean and unit variance and $\mathbf{R}_{\mathrm{MS}}^{k}$ and $\mathbf{R}_{\mathrm{BS}}$ are spatial correlation matrices for the $k$ th MS and BS, respectively.

In a rich scattering environment, the correlation for any pair of dipole elements with spacing $d_{m, n}$ can be obtained using the model in [21] and are given by the first kind Bessel function of 0 th order

$$
\rho_{m, n}=J_{0}\left(k d_{m, n}\right) .
$$

Using (5), the spatial correlation matrix for the $k$ th MS can be generated as

$$
\mathbf{R}_{\mathrm{MS}}^{k}=\left[\begin{array}{ccc}
\rho_{1,1}^{k} & \cdots & \rho_{1, M_{k}}^{k} \\
\vdots & \ddots & \vdots \\
\rho_{M_{k}, 1}^{k} & \cdots & \rho_{M_{k}, M_{k}}^{k}
\end{array}\right]
$$

In turn, the spatial correlation matrix for BS can be obtained from

$$
\mathbf{R}_{\mathrm{BS}}=\left[\begin{array}{ccc}
\rho_{1,1} & \cdots & \rho_{1, N} \\
\vdots & \ddots & \vdots \\
\rho_{N, 1} & \cdots & \rho_{N, N}
\end{array}\right] .
$$

\section{Mutual Coupling within an Array}

The interactions between the entire set of antennas within an array and scattering objects can be described by the impedance matrix $\mathbf{Z}$. For the array formed by linear parallel 
wire dipoles, the mutual impedance matrix can be obtained using electromagnetic and circuit theory described in [15, 22]

$$
\mathbf{C}=\left(Z_{A}+Z_{T}\right)\left(\mathbf{Z}+Z_{T} \mathbf{I}_{M}\right)^{-1}
$$

where $Z_{A}=73+j 42.5[\Omega]$ is the element impedance in isolation and $Z_{T}$ is impedance of the receiver at each element. It is chosen to be the complex conjugate of $Z_{A}$ to obtain the impedance match. $\mathbf{Z}$ is the mutual impedance matrix with all the diagonal elements equal to $Z_{A}+Z_{T}$; its nondiagonal elements $Z_{n m}$ are decided by the physical parameters including dipole length and the horizontal distance between the two dipoles. For a side-by-side array configuration and dipole length $l$ equals to $0.5 \lambda, Z_{n m}$ is given by $[15,18,22]$

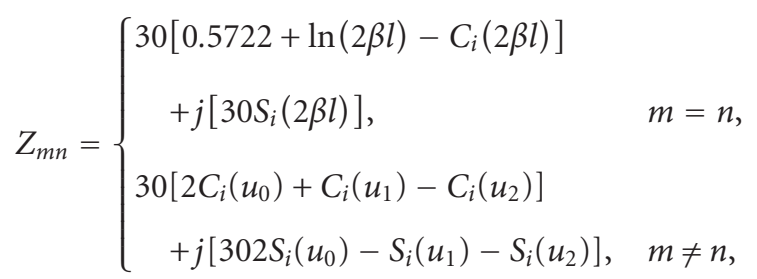

where $\beta=2 \pi / \lambda$ is the wave number and $C_{i}(u)$ and $S_{i}(u)$ are the cosine and sine integral, respectively, given as [22]

$$
\begin{aligned}
& C_{i}(u)=\int_{\infty}^{u} \frac{\cos (x)}{x} d x, \\
& S_{i}(u)=\int_{0}^{u} \frac{\sin (x)}{x} d x,
\end{aligned}
$$

and the constants are given by [18]

$$
\begin{gathered}
u_{0}=\beta d_{h}, \\
u_{1}=\beta\left(\sqrt{d_{h}^{2}+l^{2}}+l\right), \\
u_{2}=\beta\left(\sqrt{d_{h}^{2}+l^{2}}-l\right),
\end{gathered}
$$

where $d_{h}$ is the horizontal distance between the two dipole antennas.

Once the mutual coupling matrix for the transmit array at $\mathrm{BS}$ and receive arrays at MSs are obtained, we can have mutual coupling taken into account by building effective MIMO channels. As a result, the effective MIMO channel between the BS and the $k$ th MS is given as

$$
\mathbf{H}_{k}=\mathbf{C}_{\mathrm{MS}}^{k}\left(\mathbf{R}_{\mathrm{MS}}^{k}\right)^{1 / 2} \mathbf{G}_{H}^{k} \mathbf{R}_{\mathrm{BS}}^{1 / 2} \mathbf{C}_{\mathrm{BS}}
$$

\section{Impact of Mutual Coupling on the Performance of Generalized Channel Inversion}

With spatial correlation and mutual coupling taken into account, the received signal at $k$ th MS described by (2) can be rewritten as

$$
\begin{aligned}
\mathbf{y}_{k}= & \mathbf{C}_{\mathrm{MS}}^{k}\left(\mathbf{R}_{\mathrm{MS}}^{k}\right)^{1 / 2} \mathbf{G}_{H}^{k} \mathbf{R}_{\mathrm{BS}}^{1 / 2} \mathbf{C}_{\mathrm{BS}} \sum_{k=1}^{K} \mathbf{W}_{k} \mathbf{x}_{k}+\boldsymbol{\eta}_{k} \\
= & \mathbf{C}_{\mathrm{MS}}^{k}\left(\mathbf{R}_{\mathrm{MS}}^{k}\right)^{1 / 2} \mathbf{G}_{H}^{k} \mathbf{R}_{\mathrm{BS}}^{1 / 2} \mathbf{C}_{\mathrm{BS}} \mathbf{W}_{k} \mathbf{x}_{k} \\
& +\underbrace{\mathbf{C}_{\mathrm{MS}}^{k}\left(\mathbf{R}_{\mathrm{MS}}^{k}\right)^{1 / 2} \mathbf{G}_{H}^{k} \mathbf{R}_{\mathrm{BS}}^{1 / 2} \mathbf{C}_{\mathrm{BS}} \underbrace{K}_{j=1, j \neq k} \mathbf{W}_{j} \mathbf{x}_{j}+\boldsymbol{\eta}_{k} .} .
\end{aligned}
$$

Inter-user interference

The second term in (13) represents the inter-user interference. In a downlink multiuser MIMO system, the existence of inter-user interference leads to an enhanced interference and noise level, thus rendering a decreased performance. Interuser interference cancellation is thus necessary to improve operation of a multiuser MIMO system.

Inter-user interference cancellation relies on precoding techniques. To completely eliminate the inter-user interference, linear precoding techniques require that

$$
\begin{gathered}
\mathbf{C}_{\mathrm{MS}}^{i}\left(\mathbf{R}_{\mathrm{MS}}^{i}\right)^{1 / 2} \mathbf{G}_{H}^{i} \mathbf{R}_{\mathrm{BS}}^{1 / 2} \mathbf{C}_{\mathrm{BS}} \mathbf{w}_{j}=\mathbf{0}, \\
\forall i \neq j, 1 \leq i, j \leq K, \mathbf{w}_{j} \in u\left(N_{t}, M_{j}\right),
\end{gathered}
$$

where $u(a, b)$ represents the class of $a \times b$ unitary matrices, in where the $b$-tuple $\left(\mathbf{u}_{1}, \mathbf{u}_{2}, \ldots, \mathbf{u}_{b}\right)$ is orthonormal.

4.1. Interuser Interference Elimination. With spatial correlations and mutual coupling taken into account, the effective wireless links between the BS and the $K$ MSs can be represented by an overall MIMO channel, which is given as

$$
\mathbf{H}_{g}=\left[\begin{array}{llll}
\mathbf{C}_{\mathrm{MS}}^{1}\left(\mathbf{R}_{\mathrm{MS}}^{1}\right)^{1 / 2} & & & \\
& \mathbf{C}_{\mathrm{MS}}^{2}\left(\mathbf{R}_{\mathrm{MS}}^{2}\right)^{1 / 2} & & \\
& \ddots & \\
& & & \mathbf{C}_{\mathrm{MS}}^{K}\left(\mathbf{R}_{\mathrm{MS}}^{K}\right)^{1 / 2}
\end{array}\right]
$$

$$
\times\left[\begin{array}{c}
\mathbf{G}_{H}^{1} \\
\mathbf{G}_{H}^{2} \\
\vdots \\
\mathbf{G}_{H}^{K}
\end{array}\right]\left(\mathbf{R}_{\mathrm{BS}}\right)^{1 / 2} \mathbf{C}_{\mathrm{BS}} .
$$

In the generalized channel inversion scheme, the precoding matrix can be obtained by applying the minimum: meansquare error-(MMSE)-based channel inversion introduced 
in [23]. The MMSE-based generalized inversed MIMO channel can then be expressed as

$$
\mathbf{H}_{\mathrm{GCI}}=\left(\mathbf{H}_{g}^{H} \mathbf{H}_{g}+\alpha \mathbf{I}\right)^{-1} \mathbf{H}_{g}^{H}
$$

where $\alpha$ denotes the reciprocal of the ratio of total transmit energy to total noise power, as given by [23]

$$
\alpha=\frac{\sigma_{n}^{2}}{P_{t x}} \sum_{k=1}^{K} M_{k}
$$

Expression (17) shows that when the signal-to-noise ratio (SNR) is high, (16) can be approximately rewritten as

$$
\mathbf{H}_{\mathrm{GCI}}=\left(\mathbf{H}_{g}^{H} \mathbf{H}_{g}\right)^{-1} \mathbf{H}_{g}^{H} .
$$

When (18) is applied to the transmitter as the precoding matrix it can be viewed as the classical zero-forcing ( $\mathrm{ZF}$ ) filter [23]. Therefore, at high SNR, MMSE-based channel inversion is expected to have performance similar to ZFbased channel inversion.

The generalized inversed MIMO channel presented by (18) can be rewritten as a group of submatrices, which can be expressed as

$$
\mathbf{H}_{\mathrm{GCI}}=\left[\hat{\mathbf{H}}_{1}, \hat{\mathbf{H}}_{2}, \ldots, \hat{\mathbf{H}}_{K}\right]
$$

under condition

$$
\mathbf{H}_{i} \hat{\mathbf{H}}_{j}=\mathbf{0}, \quad \forall i \neq j, 1 \leq i, j \leq K
$$

To satisfy the conditions presented in (14), we need to obtain the unitary spaces for each MS. To achieve this, QR decomposition is employed over submatrices in (19). For the $j$ th MS, the QR decomposition is given as

$$
\hat{\mathbf{H}}_{j}=\widehat{\mathbf{Q}}_{j} \hat{\mathbf{R}}_{j}=\left[\hat{\mathbf{Q}}_{j}^{(1)}, \hat{\mathbf{Q}}_{j}^{(0)}\right]\left[\begin{array}{c}
\hat{\mathbf{R}}_{j}^{(1)} \\
\hat{\mathbf{R}}_{j}^{(0)}
\end{array}\right], \quad 1 \leq j \leq K,
$$

where $\hat{\mathbf{Q}}_{j}^{(1)}$ is the desired $N \times M_{j}$ unitary matrix with its columns being orthogonal unit vectors and $\hat{\mathbf{R}}_{j}^{(1)}$ is a $M_{j} \times M_{j}$ upper triangular matrix.

As a result of (20) and (21), we have

$$
\mathbf{H}_{i} \hat{\mathbf{Q}}_{j}^{(1)}=\mathbf{0}, \quad \forall i \neq j, 1 \leq i, j \leq K .
$$

To eliminate the inter-user interference, the precoding matrix for the $j$ th MS can be obtained by simply letting

$$
\mathbf{w}_{j}=\widehat{\mathbf{Q}}_{j}^{(1)} \text {. }
$$

4.2. Residual Interference Suppression. Equation (22) holds for the case when SNR is high. When the noise level is increased to a nonnegligible level, (22) is not valid any more. Consequently, it has to be rewritten as

$$
\mathbf{H}_{i} \hat{\mathbf{Q}}_{j}^{(1)} \neq \mathbf{0}, \quad \forall i \neq j, 1 \leq i, j \leq K
$$

Formula (24) indicates that the inter-user interference cannot be fully eliminated by the procedure given in (19)(23). The residual interference is due to the presence of $\alpha$ in (16). To guarantee an improved performance of the multiuser MIMO system employing generalized channel inversion scheme, an additional residual interference suppression process is required. In [24], two residual interference suppression techniques are designed under different power constraint. The one employing minimum total MSE criterion under total transmit power constraint offers a better sum rate capacity. Following the work in $[14,24]$, the residual interference filter for the $j$ th MS as $\mathbf{B}_{j}$, can be expressed as

$$
\mathbf{B}_{j}=\beta \mathbf{T}_{j}
$$

By employing the minimum total MSE criterion under total transmit power constraint, the term on the right-hand side of (25) is given as [14]

$$
\begin{gathered}
\mathbf{T}_{j}=\left(\hat{\mathbf{Q}}_{j}^{(1) H} \sum_{k=1}^{K} \mathbf{H}_{k}^{H} \mathbf{H}_{k} \hat{\mathbf{Q}}_{j}^{(1)}+\alpha \mathbf{I}\right)^{-1} \hat{\mathbf{Q}}_{j}^{(1) H} \mathbf{H}_{j}^{H} \mathbf{H}_{j} \hat{\mathbf{Q}}_{j}^{(1)}, \\
\beta=\sqrt{P_{t x}}\left[\sum_{j=1}^{K} \operatorname{Tr}\left(\mathbf{T}_{j}^{H} \mathbf{T}_{j}\right)\right]^{-1 / 2} .
\end{gathered}
$$

4.3. Impact of Mutual Coupling on Performance of Generalized Channel Inversion. It is worth noting that the inter-user elimination given by (23) and the residual interference suppression given by (26) are both unitary. By applying them to (2), the multiuser MIMO system can be decomposed into a group of point-to-point MIMO systems. As a result, the received signal at the $k$ th $\mathrm{MS}$ can be given as

$$
\mathbf{y}_{k}(n)=\mathbf{H}_{k} \hat{\mathbf{Q}}_{k}^{(1)} \mathbf{B}_{k} \mathbf{x}_{k}(n)+\mathbf{H}_{k} \sum_{j \neq k} \hat{\mathbf{Q}}_{j}^{(1)} \mathbf{B}_{j} \mathbf{x}_{j}(n)+\boldsymbol{\eta}_{k}(n) .
$$

The inter-user interference term in (27) can be neglected. As a result, the effective link between the BS and the $k$ th MS can be expressed as

$$
\mathbf{H}_{\mathrm{eff}, k}=\mathbf{H}_{k} \hat{\mathbf{Q}}_{k}^{(1)} \mathbf{B}_{k}=\mathbf{C}_{\mathrm{MS}}^{k}\left(\mathbf{R}_{\mathrm{MS}}^{k}\right)^{1 / 2} \mathbf{G}_{H}^{k} \mathbf{R}_{\mathrm{BS}}^{1 / 2} \mathbf{C}_{\mathrm{BS}} \hat{\mathbf{Q}}_{k}^{(1)} \mathbf{B}_{k} .
$$

It is assumed that $p_{k}$ is the transmit power allocated to the $k$ th MS. The capacity for the $k$ th point-to-point MIMO system can be given as

$$
C_{k}=\log _{2} \operatorname{det}\left(\mathbf{I}+\frac{p_{k}}{\sigma_{n}^{2}} \mathbf{H}_{\mathrm{eff}, k} \mathbf{H}_{\mathrm{eff}, k}^{\dagger}\right) .
$$

The sum rate capacity is then given by the sum of capacities of the $K$ point-to-point MIMO systems

$$
C_{\text {Sum }}=\sum_{k=1}^{K} \log _{2} \operatorname{det}\left(\mathbf{I}+\frac{p_{k}}{\sigma_{n}^{2}} \mathbf{H}_{\mathrm{eff}, k} \mathbf{H}_{\mathrm{eff}, k}^{\dagger}\right),
$$


when SNR is high, and (29) can be simplified to

$$
C_{k}=\log _{2} \operatorname{det}\left(\frac{p_{k}}{\sigma_{n}^{2}} \mathbf{H}_{\mathrm{eff}, k} \mathbf{H}_{\mathrm{eff}, k}^{\dagger}\right) .
$$

To include the impact of mutual coupling and spatial correlation on the sum rate capacity of the multiuser MIMO system, (12) has to be substituted into (31). The result is

$$
\begin{aligned}
C_{k}=\log _{2} \operatorname{det}( & \frac{p_{k}}{\sigma_{n}^{2}}\left(\mathbf{C}_{\mathrm{MS}}^{k}\left(\mathbf{R}_{\mathrm{MS}}^{k}\right)^{1 / 2} \mathbf{G}_{H}^{k} \mathbf{R}_{\mathrm{BS}}^{1 / 2} \mathbf{C}_{\mathrm{BS}} \hat{\mathbf{Q}}_{k}^{(1)} \mathbf{B}_{k}\right) \\
& \left.\times\left(\mathbf{C}_{\mathrm{MS}}^{k}\left(\mathbf{R}_{\mathrm{MS}}^{k}\right)^{1 / 2} \mathbf{G}_{H}^{k} \mathbf{R}_{\mathrm{BS}}^{1 / 2} \mathbf{C}_{\mathrm{BS}} \hat{\mathbf{Q}}_{k}^{(1)} \mathbf{B}_{k}\right)^{\dagger}\right) .
\end{aligned}
$$

According to (30), the sum rate capacity can be given as

$$
\begin{aligned}
C_{\text {Sum }}=\sum_{k=1}^{K} \log _{2} \operatorname{det}( & \frac{p_{k}}{\sigma_{n}^{2}}\left(\mathbf{C}_{\mathrm{MS}}^{k}\left(\mathbf{R}_{\mathrm{MS}}^{k}\right)^{1 / 2} \mathbf{G}_{H}^{k} \mathbf{R}_{\mathrm{BS}}^{1 / 2} \mathbf{C}_{\mathrm{BS}} \hat{\mathbf{Q}}_{k}^{(1)} \mathbf{B}_{k}\right) \\
& \left.\times\left(\mathbf{C}_{\mathrm{MS}}^{k}\left(\mathbf{R}_{\mathrm{MS}}^{k}\right)^{1 / 2} \mathbf{G}_{H}^{k} \mathbf{R}_{\mathrm{BS}}^{1 / 2} \mathbf{C}_{\mathrm{BS}} \hat{\mathbf{Q}}_{k}^{(1)} \mathbf{B}_{k}\right)^{\dagger}\right)
\end{aligned}
$$

Equation (32) can be further rewritten as

$$
\begin{aligned}
C_{k}=\log _{2} \operatorname{det}( & \frac{p_{k}}{\sigma_{n}^{2}}\left(\mathbf{C}_{\mathrm{MS}}^{k}\left(\mathbf{R}_{\mathrm{MS}}^{k}\right)^{1 / 2} \mathbf{G}_{H}^{k} \mathbf{R}_{\mathrm{BS}}^{1 / 2} \mathbf{C}_{\mathrm{BS}} \hat{\mathbf{Q}}_{k}^{(1)} \mathbf{B}_{k}\right) \\
& \left.\times\left(\mathbf{C}_{\mathrm{MS}}^{k}\left(\mathbf{R}_{\mathrm{MS}}^{k}\right)^{1 / 2} \mathbf{G}_{H}^{k} \mathbf{R}_{\mathrm{BS}}^{1 / 2} \mathbf{C}_{\mathrm{BS}} \hat{\mathbf{Q}}_{k}^{(1)} \mathbf{B}_{k}\right)^{\dagger}\right) \\
= & \log _{2}\left\{\operatorname { d e t } \left(\frac{p_{k}}{\sigma_{n}^{2}}\left(\left(\mathbf{R}_{\mathrm{MS}}^{k}\right)^{1 / 2} \mathbf{G}_{H}^{k} \mathbf{R}_{\mathrm{BS}}^{1 / 2} \mathbf{C}_{\mathrm{BS}} \hat{\mathbf{Q}}_{k}^{(1)} \mathbf{B}_{k}\right)\right.\right. \\
\times & \left.\left(\left(\mathbf{R}_{\mathrm{MS}}^{k}\right)^{1 / 2} \mathbf{G}_{\mathrm{H}}^{k} \mathbf{R}_{\mathrm{BS}}^{1 / 2} \mathbf{C}_{\mathrm{BS}} \hat{\mathbf{Q}}_{k}^{(1)} \mathbf{B}_{k}\right)^{\dagger}\right) \\
\times & \left.\operatorname{det}\left(\mathbf{C}_{\mathrm{MS}}^{k} \mathrm{C}_{\mathrm{MS}}^{k}\right)\right\} \\
=\log _{2} \operatorname{det}( & \frac{p_{k}}{\sigma_{n}^{2}}\left(\mathbf{R}_{\mathrm{MS}}^{k}\right)^{1 / 2} \mathbf{G}_{H}^{k} \mathbf{R}_{\mathrm{BS}}^{1 / 2} \mathbf{C}_{\mathrm{BS}} \hat{\mathbf{Q}}_{k}^{(1)} \mathbf{B}_{k} \\
& \left.\times\left(\left(\mathbf{R}_{\mathrm{MS}}^{k}\right)^{1 / 2} \mathbf{G}_{H}^{k} \mathbf{R}_{\mathrm{BS}}^{1 / 2} \mathbf{C}_{\mathrm{BS}} \hat{\mathbf{Q}}_{k}^{(1)} \mathbf{B}_{k}\right)^{\dagger}\right) \\
+ & \log _{2} \operatorname{det}\left(\mathbf{C}_{\mathrm{MS}}^{k} \mathbf{C}_{\mathrm{MS}}^{k}{ }^{\dagger}\right) .
\end{aligned}
$$

Equation (34) shows there are two terms contributing to the capacity of the $k$ th MS. The first term represents the capacity of the $k$ th MS without the mutual coupling taken into account at MS. The second term denotes contribution of the mutual coupling at MSs to the sum rate capacity. By substituting (34) into (33), the sum rate capacity is given as

$$
\begin{aligned}
C_{\text {sum }}= & \sum_{k=1}^{K} \log _{2} \operatorname{det}\left(\frac{p_{k}}{\sigma_{n}^{2}}\left(\mathbf{R}_{\mathrm{MS}}^{k}\right)^{1 / 2} \mathbf{G}_{H}^{k} \mathbf{R}_{\mathrm{BS}}^{1 / 2} \mathbf{C}_{\mathrm{BS}} \hat{\mathbf{Q}}_{k}^{(1)} \mathbf{B}_{k}\right. \\
& \left.\times\left(\left(\mathbf{R}_{\mathrm{MS}}^{k}\right)^{1 / 2} \mathbf{G}_{H}^{k} \mathbf{R}_{\mathrm{BS}}^{1 / 2} \mathbf{C}_{\mathrm{BS}} \hat{\mathbf{Q}}_{k}^{(1)} \mathbf{B}_{k}\right)^{\dagger}\right) \\
& +\sum_{k=1}^{K} \log _{2} \operatorname{det}\left(\mathbf{C}_{\mathrm{MS}}^{k} \mathbf{C}_{\mathrm{MS}}^{k}{ }^{\dagger}\right) .
\end{aligned}
$$

Therefore, the impact of mutual coupling at MSs on the sum rate capacity can be expressed as

$$
\Delta \mathrm{C}_{\text {mutual coupling }}=\sum_{k=1}^{K} \log _{2} \operatorname{det}\left(\mathrm{C}_{\mathrm{MS}}^{k} \mathrm{C}_{\mathrm{MS}}{ }^{\dagger}\right) \text {. }
$$

As seen from (36), the impact of mutual coupling at MSs on sum rate capacity depends on the coupling matrices at the MSs. If the product of the determinants of the mutual coupling matrices is larger than one, the effect of mutual coupling on capacity is positive. Otherwise, it is negative. This can also be explained from the view of effective spatial correlation. As shown by (12), with mutual coupling taken into account, the effective spatial correlations at BS and the $k$ th MS can be given as

$$
\begin{gathered}
\mathbf{R}_{k, \mathrm{eff}}^{1 / 2}=\mathbf{C}_{\mathrm{MS}}^{k}\left(\mathbf{R}_{\mathrm{MS}}^{k}\right)^{1 / 2}, \\
\mathbf{R}_{\mathrm{BS}, \mathrm{eff}}^{1 / 2}=\mathbf{R}_{\mathrm{BS}}^{1 / 2} \mathbf{C}_{\mathrm{BS}}
\end{gathered}
$$

These effective spatial correlation matrices represent mutual coupling modulated spatial correlations. If the existence of the mutual coupling results in larger effective spatial correlations in an antenna array, it has negative impact on the sum rate capacity of the multiuser MIMO system.

\section{Numerical Results}

To investigate the impact of antenna mutual coupling on the performance of a multiuser MIMO system employing generalized channel inversion, the Monto-Carlo simulations are performed. In the assumed system, the BS is equipped with 6 transmit dipole antennas, and there are 2 MSs each having 3 receive dipole antennas. Transmit power is equally allocated to the 2 MSs. Both for BS and MSs, the dipole antennas are assumed to be $0.5 \lambda$ in length. This system is referred to as $2 \times(3 \times 6)$ system.

The numerical results are divided into three groups, in which only transmit correlation and mutual coupling are considered, only receive correlation and mutual coupling are considered, and where both transmit and receive correlation and mutual coupling are considered. In the simulations, the unit for interelement spacing is one wavelength and denoted by $\lambda$. In the following figures, each of the presented (point) results is obtained from 5000 independent simulation runs. 


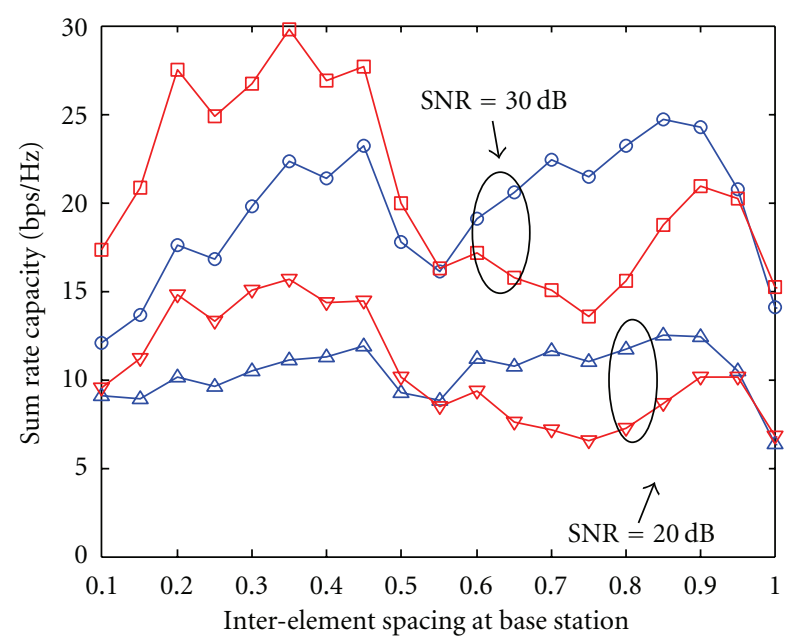

$\triangle$ w/o mutual coupling SNR $=20 \mathrm{~dB}$

$\rightarrow$ With mutual coupling SNR $=20 \mathrm{~dB}$

- w/o mutual coupling $\mathrm{SNR}=30 \mathrm{~dB}$

$\square$ With mutual coupling SNR $=30 \mathrm{~dB}$

Figure 1: Sum rate capacity with regards to interelement spacing at BS for a $2 \times(3 \times 6)$ multiuser MIMO system with and without mutual coupling taken into account.

5.1. Impact of Mutual Coupling at BS on the System Performance. In the first set of simulations, spatial correlation and mutual coupling at BS are considered. We assume that there are no spatial correlations or mutual coupling effects at the MSs. The simulations are designed to show the impact of mutual coupling at the BS side on the sum rate capacity of a multiuser MIMO system. Figure 1 shows the sum rate capacity for a $2 \times(3 \times 6)$ multiuser MIMO system with the varying interelement spacing at the BS.

It can be seen from results presented in Figure 1 that without mutual coupling (where only spatial correlation is considered) the system achieves " $\mathrm{M}$ "-looking sum rate capacity plots for SNR $=20 \mathrm{~dB}$ and SNR $=30 \mathrm{~dB}$. Both plots peak at BS interelement spacing of $0.45 \lambda$ and $0.85 \lambda$ with the $0.85 \lambda$ interelement spacing offering a larger sum rate capacity. As observed, the sum rate capacity plots for the $2 \times(3 \times 6)$ multiuser MIMO system with mutual coupling taken into account also show a M'-looking. They peak at BS interelement spacing of $0.35 \lambda$ and $0.9 \lambda$ with the $0.35 \lambda$ interelement spacing offering a larger sum rate capacity. Comparing the plots of sum rate capacity with and without mutual coupling taken into account, it can be seen from Figure 1 that their values cross when the interelement spacing is $0.55 \lambda$, for both $\mathrm{SNR}=20 \mathrm{~dB}$ and $\mathrm{SNR}=30 \mathrm{~dB}$. Also, when the interelement spacing is smaller than $0.55 \lambda$ the presence of mutual coupling results in an increased sum rate capacity. Therefore, mutual coupling has a positive effect on the performance of the system in terms of sum rate capacity. When the interelement spacing is larger than $0.55 \lambda$, the presence of mutual coupling results in a decreased sum rate capacity. Therefore, in this case mutual coupling has a negative effect on the performance of the system in terms of sum

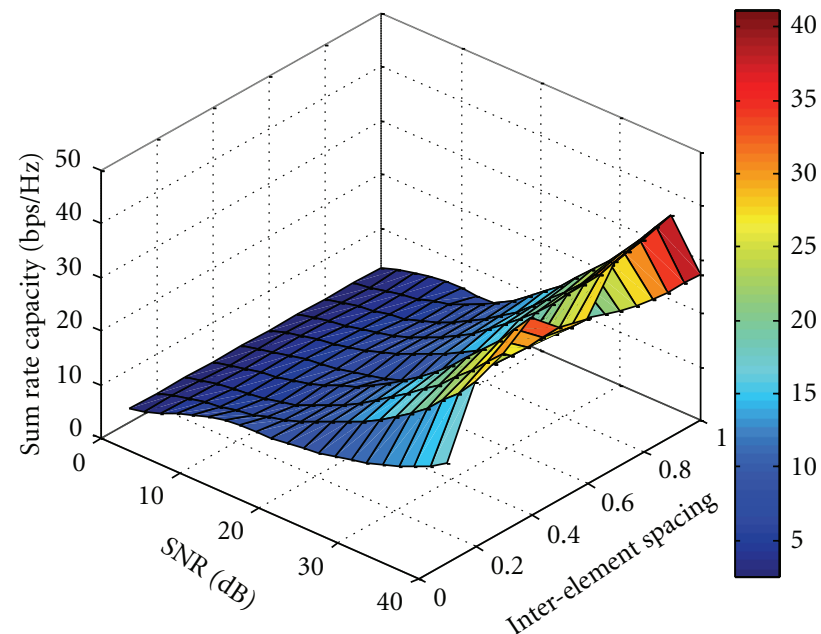

FIGURE 2: Surface of sum rate capacity with regard to interelement spacing at BS and SNR for a $2 \times(3 \times 6)$ multiuser MIMO system without mutual coupling taken into account.

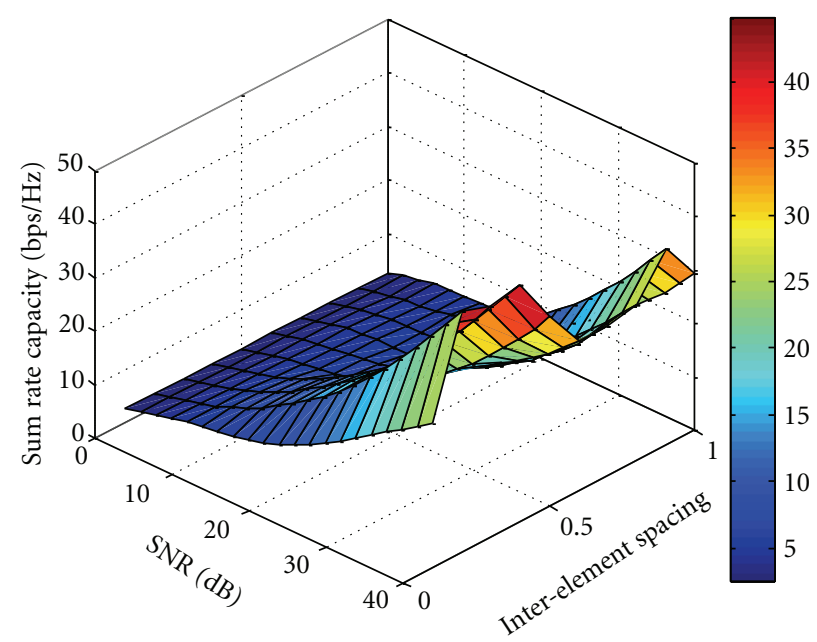

FIGURE 3: Surface of sum rate capacity with regard to interelement spacing at BS and SNR for a $2 \times(3 \times 6)$ multiuser MIMO system with mutual coupling taken into account.

rate capacity. When the interelement spacing approaches $1.0 \lambda$, the effect of mutual coupling becomes negligible.

Figures 2 and 3 offer an extension of results earlier shown in Figure 1 for an SNR range of 0 to $40 \mathrm{~dB}$.

Cases of with and without mutual coupling taken into account are considered. In both cases, the sum rate capacity is shown with respect to SNR and interelement spacing at the BS. The peaks between $0.2-0.4 \lambda$ and $0.7-0.9 \lambda$ become more visible as SNR increases. In Figure 2, when mutual coupling is not considered, higher capacity is achieved for interelement spacing ranging from $0.8 \lambda$ to $0.9 \lambda$, whereas in Figure 3, with mutual coupling, higher capacity is achieved for lower interelement spacing ranging from $0.3 \lambda$ to $0.4 \lambda$.

5.2. Impact of Mutual Coupling at MSs on the System Performance. In the second set of simulations, spatial correlation 


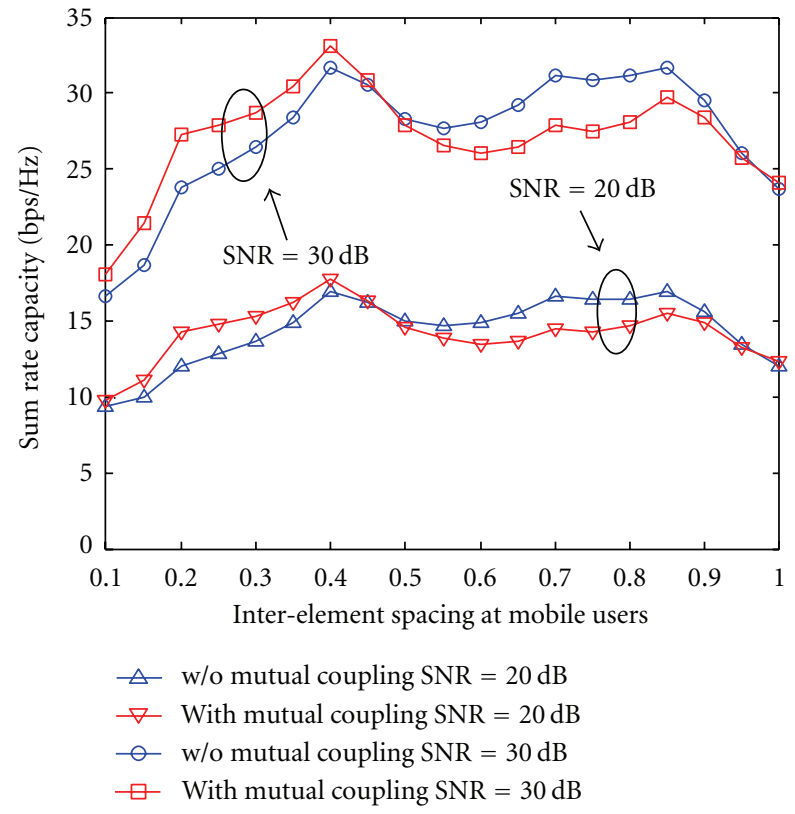

FIGURE 4: Sum rate capacity with regard to interelement spacing at MSs for a $2 \times(3 \times 6)$ multiuser MIMO system with and without mutual coupling taken into account.

and mutual coupling at MSs are considered, by varying the interelement spacing at the MSs. It is postulated that the receive array at each of the MSs has the same interelement spacing and there are no spatial correlation and mutual coupling effects at the BS. This group of simulations is designed to investigate the impact of mutual coupling at MSs' side on the sum rate capacity of a multiuser MIMO system.

Figure 4 presents the sum rate capacity for a $2 \times(3 \times 6)$ multiuser MIMO system with varying interelement spacing at MSs.

The presented results show a similar trend to the results given in Figure 1. In particular, the plots of sum rate capacity with and without mutual coupling for different SNRs show exactly the same trends. The peak values take place for interelement spacing of $0.4 \lambda$ and the range from $0.7 \lambda$ to $0.85 \lambda$. The plots of sum rate capacity for the cases with and without mutual coupling cross when the interelement spacing equals approximately $0.45 \lambda$. As shown in Figure 4, when interelement spacing is smaller than $0.45 \lambda$, the existence of mutual coupling results in an increased sum rate capacity. Therefore, mutual coupling has a positive effect on the performance of the system in terms of sum rate capacity. When interelement spacing is larger than $0.45 \lambda$, the presence of mutual coupling actually results in a decreased sum rate capacity. In this case, antenna mutual coupling has a negative effect on the performance of the system in terms of sum rate capacity. When antenna interelement spacing approaches $1.0 \lambda$, the difference between results with and without mutual coupling becomes negligible.

Figures 5 and 6 show an extension of results presented in Figure 4 for an SNR range of 0 to $40 \mathrm{~dB}$, for the cases of with and without mutual coupling taken into account. The

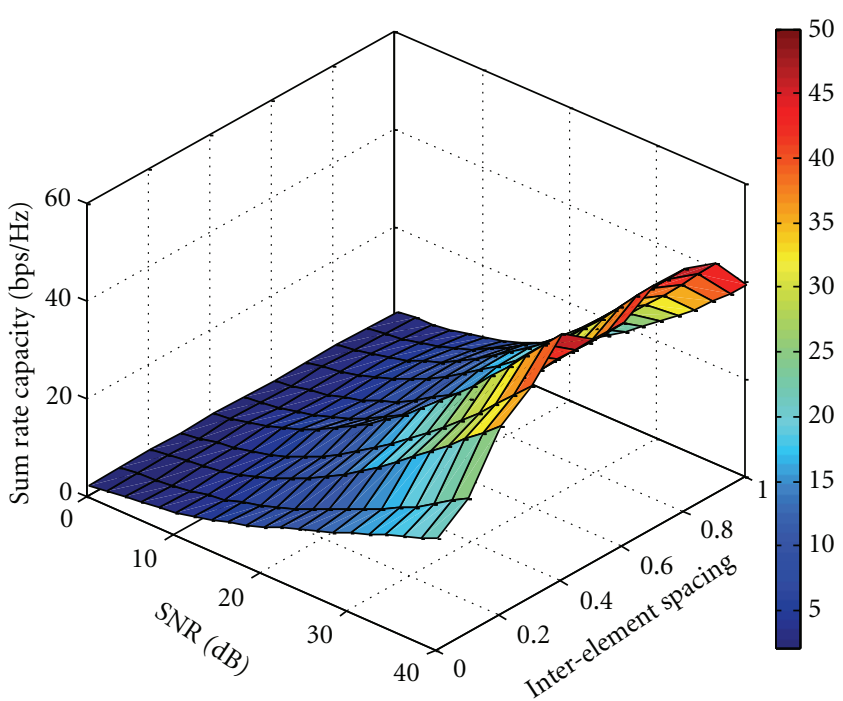

FIGURE 5: Surface of sum rate capacity with regard to interelement spacing at MSs and SNR for a $2 \times(3 \times 6)$ multiuser MIMO system without mutual coupling taken into account.

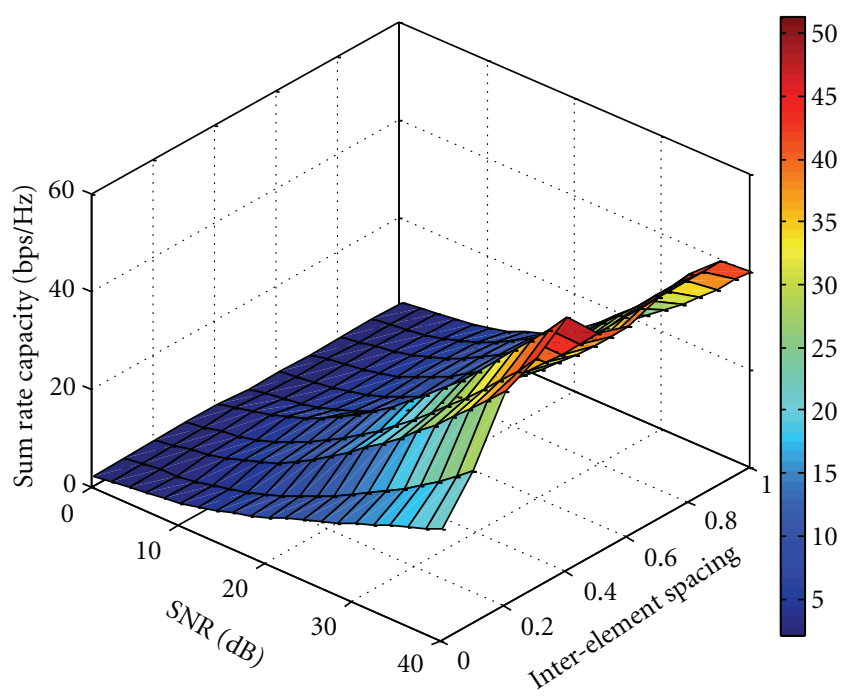

FIGURE 6: Surface of sum rate capacity with regard to interelement spacing at MSs and SNR for a $2 \times(3 \times 6)$ multiuser MIMO system with mutual coupling taken into account.

sum rate capacity is shown with respect to both SNR and interelement spacing at the MSs. The peak values occurring for the interelement spacing between $0.35-0.45 \lambda$ and $0.7-$ $0.85 \lambda$ become more visible as SNR increases.

In Figure 5, when mutual coupling is not considered, higher capacity is achieved for interelement spacing ranging from $0.8 \lambda$ to $0.85 \lambda$, whereas in Figure 6 , with mutual coupling taken into account, higher capacity is achieved for lower interelement spacing of $0.4 \lambda$.

5.3. Impact of Mutual Coupling at Both BS and MSs on the System Performance. In the third set of simulations, spatial correlations and mutual coupling at both BS and MSs are 


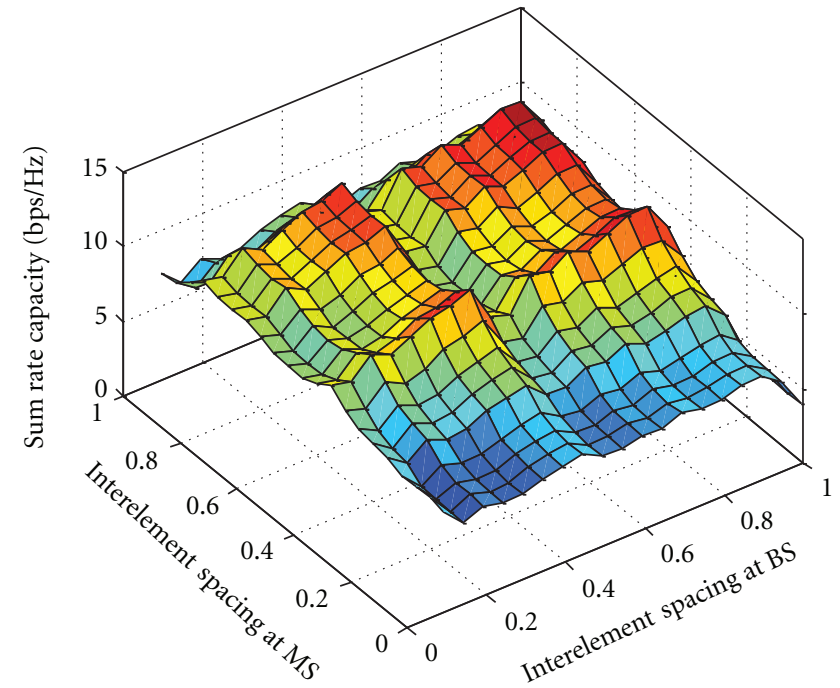

(a)

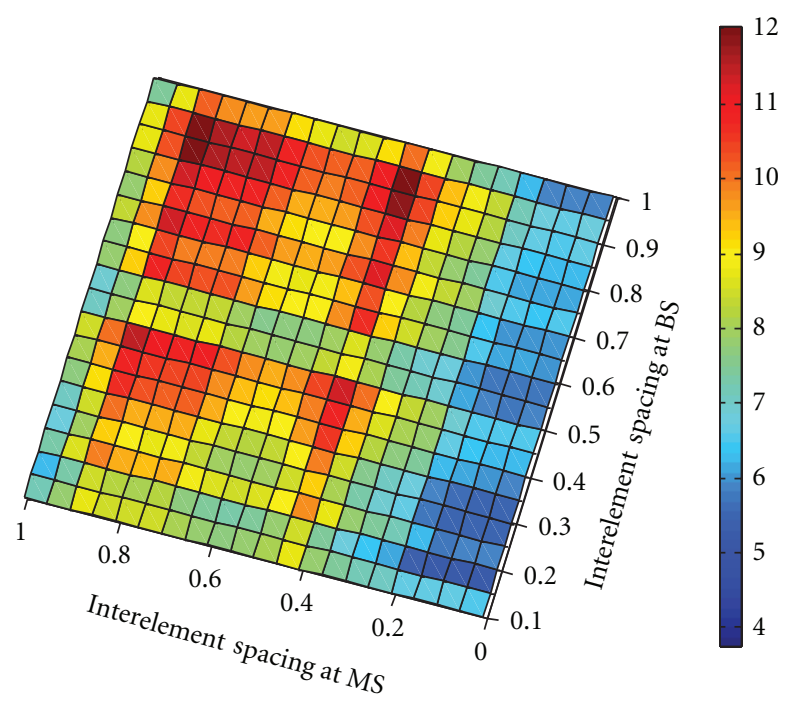

(b)

FIGURE 7: Surface of sum rate capacity with transmit and receive spatial correlation only for a $2 \times(3 \times 6)$ multiuser MIMO system at SNR $=$ $20 \mathrm{~dB}$.

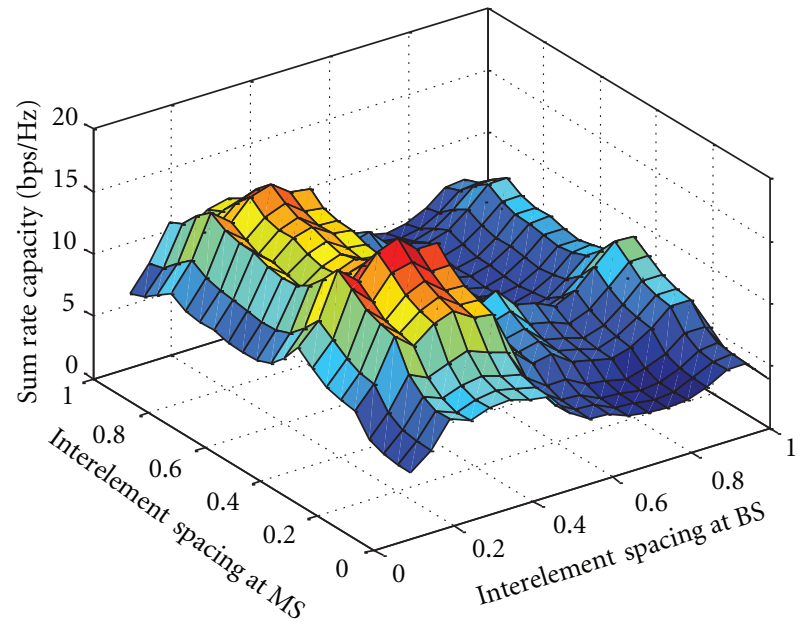

(a)

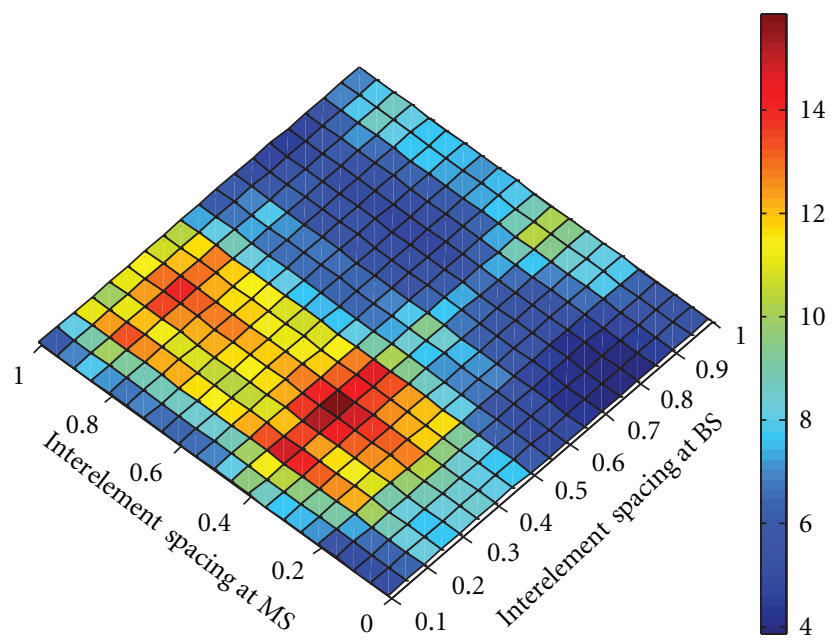

(b)

FIGURE 8: Surface of sum rate capacity with transmit and receive spatial correlation and mutual coupling for a $2 \times(3 \times 6)$ multiuser MIMO system at $\mathrm{SNR}=20 \mathrm{~dB}$.

considered, by varying the interelement spacing at the BS and MSs. We assume that the receive array at each of the MSs has the same interelement spacing. The SNRs for both cases are fixed to $20 \mathrm{~dB}$. This group of simulations are designed to reveal the joint impact of mutual coupling at both the BS and MSs on the sum rate capacity of a multiuser MIMO system.

Figure 7 shows the 2-diemnsional representation of sum rate capacity with spatial correlations at BS and MSs, where mutual coupling is not taken into account.

As expected from the previous simulations, the results in Figure 7 show four dominant peaks on the surface of sum rate capacity. These include the case when BS interelement spacing is $0.45 \lambda$ and MSs antenna interelement spacing is
$0.4 \lambda$, BS antenna interelement spacing is $0.45 \lambda$ and MSs interelement spacing is $0.9 \lambda$, BS interelement spacing is $0.9 \lambda$ with MSs interelement spacing of $0.4 \lambda$, and BS interelement spacing of $0.9 \lambda$ and MSs interelement spacing of $0.9 \lambda$. Among them, the peak located at BS antenna interelement spacing $0.9 \lambda$ with MSs antenna interelement spacing $0.9 \lambda$ is the most significant one.

Figure 8 shows the 2-dimensional representation of sum rate capacity with spatial correlations and mutual coupling taken into account at BS and MSs.

As expected from the previous simulations, the results presented in Figure 8 indicate two dominant peaks on the surface of sum rate capacity. These include the cases of BS 
interelement spacing of $0.45 \lambda$ and $0.4 \lambda$ at MSs, and BS interelement spacing of $0.45 \lambda$ and $0.85 \lambda$ at MSs, respectively. The peak located at interelement spacing of $0.45 \lambda$ at BS and $0.4 \lambda$ at MSs is the most significant one.

\section{Conclusions}

The paper has reported investigations into the performance of a multiuser MIMO system employing generalized channel inversion scheme. The investigations have taken into account spatial correlation and antenna mutual coupling effects which are present in compact array antennas. The undertaken theoretical analysis has shown that the presence of antenna mutual coupling may have positive impact on the performance of the multiuser MIMO system in terms of sum rate capacity. The simulation results have confirmed these theoretical findings by showing that when the antenna interelement spacing at BS is smaller than $0.55 \lambda$ and at MSs is less than $0.4 \lambda$, the existence of mutual coupling results in an increased sum rate capacity. The numerical results have also indicated that without mutual coupling taken into account, the system configuration with a BS antenna interelement spacing and MSs interelement spacing of $0.9 \lambda$ achieves the most significant sum rate capacity. However, this leads to a noncompact transceiver design. For the compact transceiver design, the presence of mutual coupling is beneficial in terms of an increased sum rate capacity when at a BS the antenna interelement spacing is $0.45 \lambda$ and $0.4 \lambda$ at MSs.

\section{References}

[1] G. J. Foschini and M. J. Gans, "On limits of wireless communications in a fading environment when using multiple antennas," Wireless Personal Communications, vol. 6, no. 3, pp. 311-335, 1998.

[2] E. Telatar, "Capacity of multi-antenna Gaussian channels," European Transactions on Telecommunications, vol. 10, no. 6, pp. 585-595, 1999.

[3] 3GPP TS, "LTE physical layer-general description," 36.201v1.0.0, http://www.3gpp.org/ftp/Specs/archive/36\%5Fseries/ 36.201 .

[4] 3GPP TS, "Physical channels and modulation," http://www .3gpp.org/ftp/Specs/archive/36\%5Fseries/36.211.

[5] D. Gesbert, M. Kountouris, R. W. Heath jr., C. B. Chae, and T. Sälzer, "Shifting the MIMO Paradigm," Signal Processing Magazine, vol. 24, no. 5, pp. 36-46, 2007.

[6] G. Caire and S. Shamai, "On the achievable throughput of a multiantenna Gaussian broadcast channel," IEEE Transactions on Information Theory, vol. 43, no. 7, pp. 1691-1706, 2006.

[7] P. Viswanath and D. N. C. Tse, "Sum capacity of the vector Gaussian broadcast channel," IEEE Transactions on Information Theory, vol. 49, no. 8, pp. 1912-1921, 2003.

[8] H. Weingarten, Y. Steinberg, and S. Shamai, "The capacity region of the Gaussian multiple-input multiple-output broadcast channel," IEEE Transactions on Information Theory, vol. 52, no. 9, pp. 3936-3964, 2006.

[9] M. H. M. Costa, "Writing on dirty paper," IEEE Transactions on Information Theory, vol. IT-29, no. 3, pp. 439-441, 1983.

[10] W. Yu and J. M. Cioffi, "Trellis precoding for the broadcast channel," in Proceedings of the IEEE Global Telecommunicatins Conference (GLOBECOM '01), vol. 2, pp. 1344-1348, November 2001.
[11] Q. H. Spencer, A. L. Swindlehurst, and M. Haardt, "Zeroforcing methods for downlink spatial multiplexing in multiuser MIMO channels," IEEE Transactions on Information Theory, vol. 42, no. 3, pp. 461-471, 2004.

[12] S. Shim, J. S. Kwak, R. W. Heath, and J. G. Andrews, "Block diagonalization for multi-user MIMO with other-cell interference," IEEE Transactions on Wireless Communications, vol. 7, no. 7, Article ID 4570233, pp. 2671-2681, 2008.

[13] F. Wang, M. E. Bialkowski, and X. Liu, "Performance of block diagonalization broadcasting scheme for multiuser mimo system operating in presence of spatial correlation and mutual coupling," International Journal of Communications, Network and System Science, vol. 3, no. 3, pp. 266-272, 2010.

[14] H. Sung, S. R. Lee, and I. Lee, "Generalized channel inversion methods for multiuser MIMO systems," IEEE Transactions on Communications, vol. 57, no. 11, Article ID 5336870, pp. 34893499, 2009.

[15] S. Durrani and M. E. Bialkowski, "Effect of mutual coupling on the interference rejection capabilities of linear and circular arrays in CDMA systems," IEEE Transactions on Antennas and Propagation, vol. 52, no. 4, pp. 1130-1134, 2004.

[16] H. S. Lui and H. T. Hui, "Mutual coupling compensation of compact antenna array for direction-of-arrivals estimations," in Proceedings of the 4th International Conference on Signal Processing and Communication Systems (ICSPCS'10), Gold Coast, Australia, 2010.

[17] T. Svantesson and A. Ranheim, "Mutual coupling effects on the capacity of multielement antenna systems," in Proceedings of the IEEE Interntional Conference on Acoustics, Speech, and Signal Processing (ICASSP '01), pp. 2485-2488, May 2001.

[18] M. E. Bialkowski, P. Uthansakul, K. Bialkowski, and S. Durrani, "Investigating the performance of mimo systems from an electromagnetic perspective," Microwave and Optical Technology Letters, vol. 48, no. 7, pp. 1233-1238, 2006.

[19] P. N. Fletcher, M. Dean, and A. R. Nix, "Mutual coupling in multi-element array antennas and its influence on MIMO channel capacity," Electronics Letters, vol. 39, no. 4, pp. 342$344,2003$.

[20] F. Wang and M. E. Bialkowski, "Performance of succesive broadcasting scheme for a multiuser dowlink MIMO system operating under mutual coupling conditions," in 4th International Conference on Signal Processing and Communication Systems, (ICSPCS '10), Gold Coast, Australia, 2010.

[21] W. C. Jakes, Microwave Mobile Communications, John Wiley \& Sons, New York, NY, USA, 1974.

[22] C. A. Balanis, Antenna Theory: Analysis and Design, Wiley, New York, NY, USA, 2nd edition, 1997.

[23] C. B. Peel, B. M. Hochwald, and A. L. Swindlehurst, "A vector-perturbation technique for near-capacity multiantenna multiuser communication, part I: channel inversion and regularization," IEEE Transactions on Communications, vol. 53, no. 1, pp. 195-202, 2005.

[24] H. Lee, K. Lee, B. M. Hochwald, and I. Lee, "Regularized channel inversion for multiple-antenna users in multiuser MIMO downlink," in IEEE International Conference on Communications (ICC '08), pp. 3501-3505, chn, May 2008. 

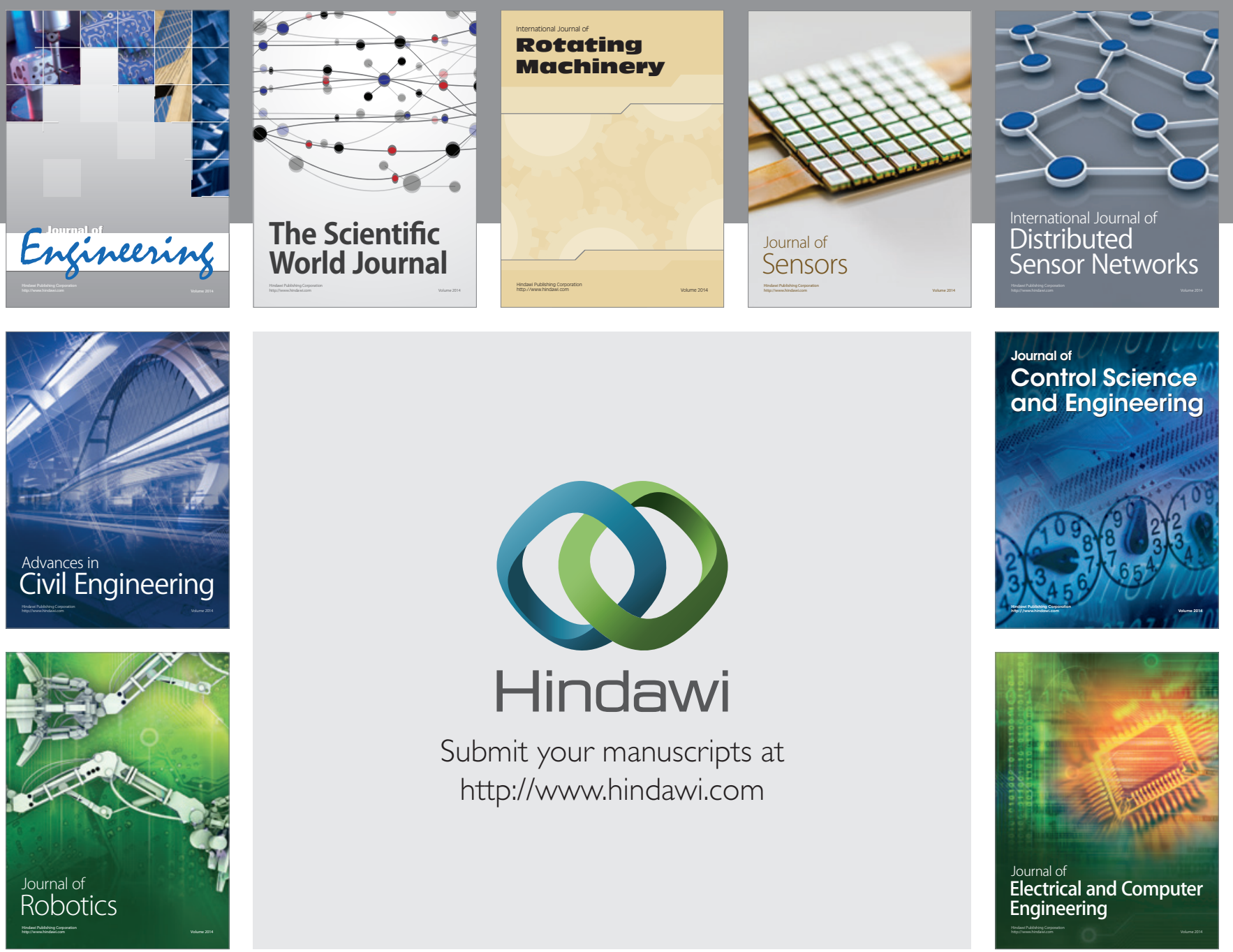

Submit your manuscripts at

http://www.hindawi.com
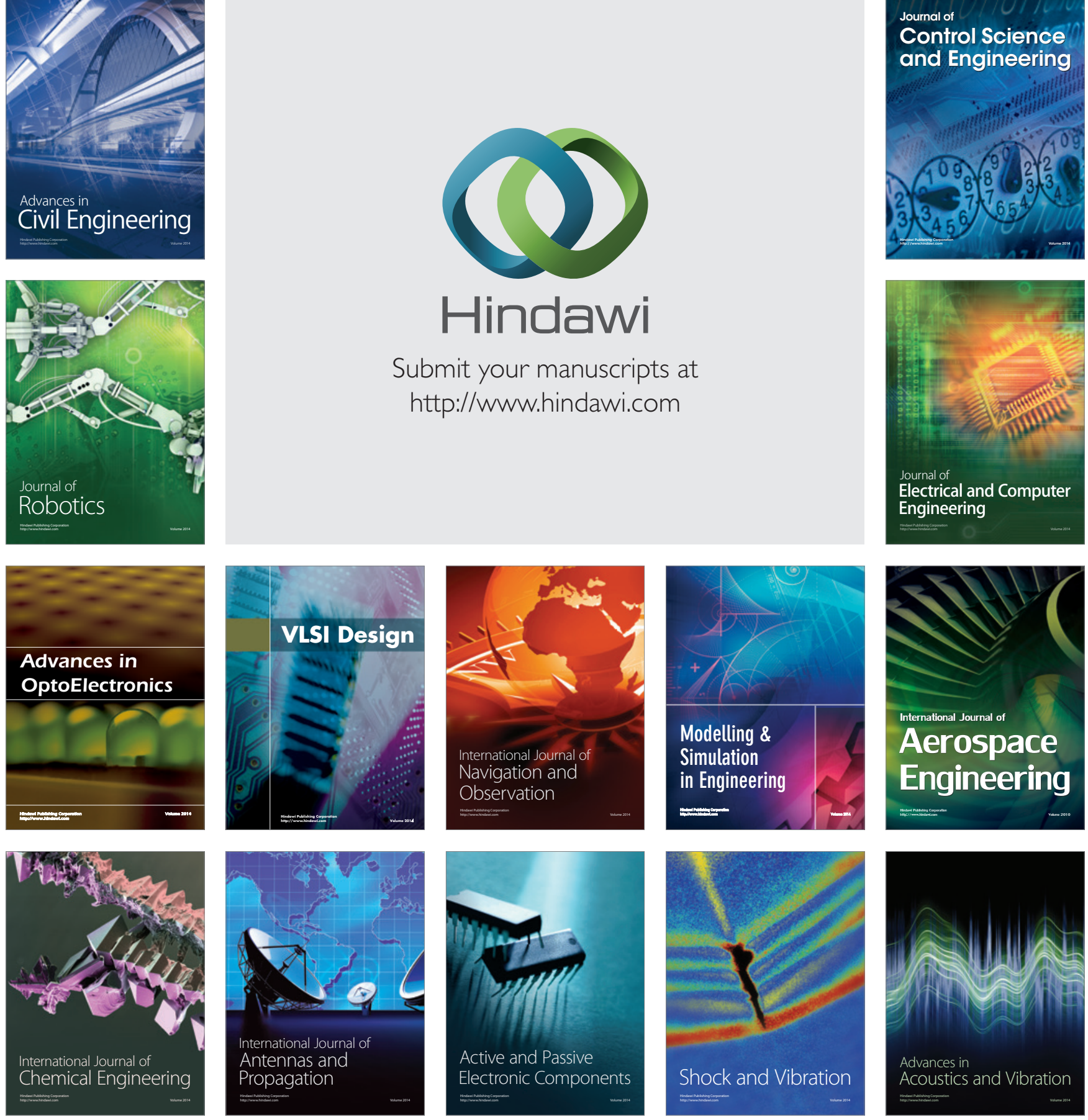